\title{
THE MULTI-BAND STUDY OF THE ENVIRONMENT OF THE RC J0311+0507 RADIO GALAXY: A STEP FORWARD TO UNDERSTAND MASSIVE STELLAR SYSTEM FORMATION AT $Z>4$
}

\author{
Yu.N. Parijskij ${ }^{1}$, O.P. Zhelenkova ${ }^{2}$, P. Thomasson ${ }^{3,4}$, A.I. Kopylov ${ }^{2}$,
} A.V. Temirova ${ }^{1}$, I.V. Sokolov ${ }^{5}$, V.N. Komarova ${ }^{2}$ and O.J.A. Bravo Calle ${ }^{6}$

\begin{abstract}
The radio galaxy RC J0311+0507 was investigated in the "Big Trio" project, which aims to search for distant radio galaxies. Optical spectroscopy performed at the 6-m telescope measured its redshift as 4.514. The source is one of the most luminous objects in the high redshift Universe having $\mathrm{L} \approx 3 \times 10^{29} \mathrm{~W} \mathrm{~Hz}^{-1}$ at $500 \mathrm{MHz}$, that suggests the presence of a super massive black hole with a mass of about $10^{10} M_{\odot}$ inside the parent galaxy. Mechanisms of the formation of galaxies with black holes of such masses in the early stage of the Universe is not yet clear. Based on a collection of deep images taken in 10 optical and infrared bands we started a photometric study of the environment of this radio galaxy to detect possible neighborhood.
\end{abstract}

\section{Discovery of the distant powerful radio galaxy}

RC J0311+0507 was recognized as having an Ultra Steep Spectrum (USS) in the frequency range $365-4850 \mathrm{MHz}\left(\alpha=-1.31, \mathrm{~S} \propto \nu^{\alpha}\right)$ in the early stages of the "Big Trio" survey project (Goss et al. 1992; Parijskij et al. 1996; Parijskij et al. 2000). The project, aimed at searching and studying very distant radio galaxies, is composed by: the RATAN-600 radio telescope as the survey instrument, the VLA radio telescope as the imaging instrument, and the 6-m optical telescope of the Special

\footnotetext{
1 Saint-Petersburg Branch of SAO RAS, Russia; e-mail: unipar@rambler.ru

2 Special Astrophysical Observatory of RAS, Russia; e-mail: zhe@sao.ru

3 Jodrell Bank Centre for Astrophysics, UK; e-mail: pt@jb.man.ac.uk

${ }^{4}$ Institute for Radio Astronomy and Space Research, New Zealand

${ }^{5}$ Institute of Astronomy RAS, Russia; e-mail: ilia333@land.ru

6 Saint Petersburg State University, Russia
} 
Astrophysical Observatory of the Russian Academy of Sciences (SAO RAS) for spectroscopy. The only known object close to the position of was the radio galaxy $4 \mathrm{C}+04.11$, and VLA images of it suggested an asymmetric double structure. Optical photometric and spectroscopic observations with the 6 - $m$ telescope allowed the identification of its host galaxy $(\mathrm{R}=22.8)$ and the measure of its redshift. Indeed, its spectrum showed the presence of a strong emission line at $6703 \AA$ which was interpreted as $L y \alpha$ at at a redshift of $z=4.514$ (Kopylov et al. 2006).

New MERLIN and EVN maps of this object, at $1658 \mathrm{MHz}$ and $4999 \mathrm{MHz}$ respectively, with an order of magnitude better resolution than those of the VLA have shown the multi-component structure and overall curved structure of the radio source (Parijskij et al. 2010). The extent of RC J0311 + $0507\left(\sim 2.8^{\prime \prime}\right)$ appears to be approximately the same as that of the optically visible galaxy. A multicomponent structure for a radio galaxy is not a very rare phenomenon, although in the case of RC J0311 + 0507, the radio luminosity of each components appears to be greater than $10^{28} \mathrm{~W} \mathrm{~Hz}^{-1}$ at $500 \mathrm{MHz}$, which is comparable with the luminosities of the main lobes of the most powerful FRII radio galaxies. The precise nature of the components is not clear, but it is possible that nuclear source activity has been stimulated by multi-merging effects and/or that the jet is propagating through and interacting with a denser environment.

An estimation of the black hole (BH) mass in RC J0311 + 0507 can be made from the correlation between the $\mathrm{BH}$ mass and the total and core radio luminosities (Franceschini et al. 1998). The derived value seems to indicate that $\mathrm{RC} \mathrm{J0311}+0507$ is an object with a supermassive black hole (SMBH) with a mass $\approx 10^{10} M_{\odot}$.

\section{Photometric study of the RC J0311+0507 environment}

We started the photometric study of the RC J0311 + 0507 environment using the available optical and infrared data. Deep photometric images include: the 6-m SAO RAS telescope observations in the B, V, R, I bands and in the SED607, SED665 and SED707 intermediate-band filters (20-30 min exposures at 1.2-2 $2^{\prime \prime}$; the 3.8-m UKIRT telescope $\mathrm{K}$ band observations (100 min exposure and $0.7-1.1^{\prime \prime}$ seeing); the 8-m SUBARU telescope observations from the SMOKA archive in B, R, V bands and NL671 narrow-band filter (30, 60, 48 and $160 \mathrm{~min}$, respectively, with $0.3-0.4^{\prime \prime}$ seeing); images in 3.6 and $4.5 \mu \mathrm{k}$ bands (30 and $45 \mathrm{~min}$ exposures respectively) from the Spitzer Heritage Archive.

All the available data were partially processed or reprocessed in a uniform manner using the ESO-MIDAS astronomical data reduction system and the Subaru Data Reduction and Analysis (SuperCam) package for the reduction of the data acquired at that telescope. Figures 1 and 2 resulting reprocessed V, R, I, SED607 and SED655 band images taken at the 6-m SAO RAS telescope and the processed NL671 narrow-band image taken at with SUBARU.

The estimation, obtained by the sum of frames, shows a slight increase in the surface density of objects around the radio source. This may indicate the presence 

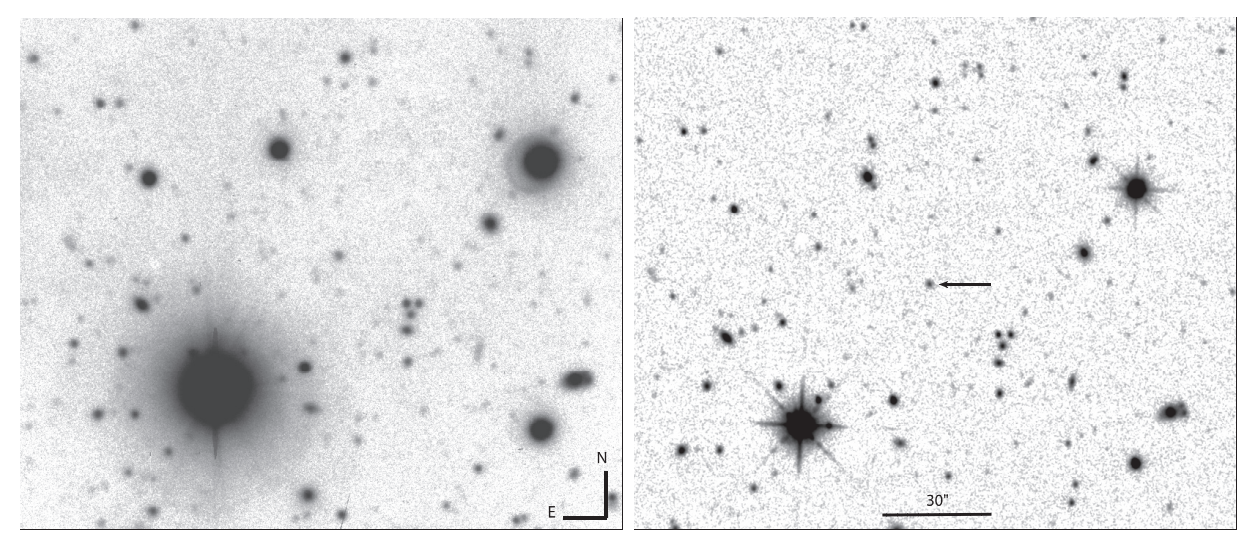

Fig. 1. Left: the sum of the R, I, SED665, SED707 images obtained with the 6-m SAO RAS telescope; right: the UKIRT K-band image. An arrow marks the host galaxy location.
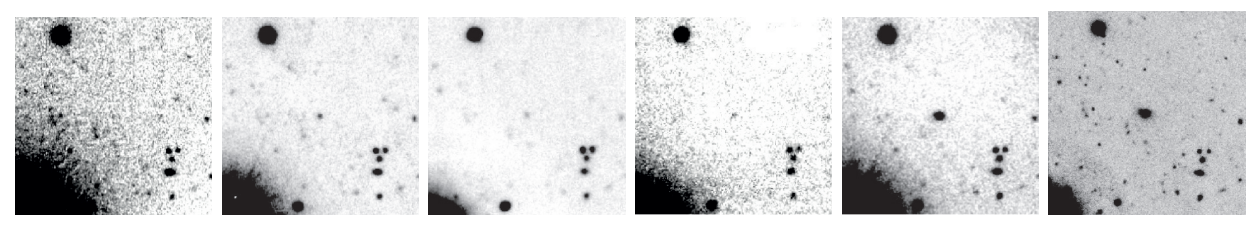

Fig. 2. The 6-m SAO RAS telescope images, obtained with the SCORPIO reducer, left to right V, R, I, SED607 and SED655 bands. The last image to the right is the NL671 narrow-band image taken at the 8-m SUBARU telescope. The host galaxy of the RC J0311 + 0507 locates in the center of images.

of closely located to RC J0311 + 0507 objects. The SED665 and NL671 narrowband images show an extended $L y \alpha$-envelope (see the last two images in Fig. 2).

\section{Conclusion}

RC J0311 + 0507 appears to be a remarkable object, its study and of other similar systems with SMBH of about $10^{10} M_{\odot}$ at redshifts greater than 4 could help to better understand their formation. We here reported on the first results of our multi wavelength photometric approach to study the environment of the radio galaxy RC J0311 + 0507. We plan a further study using known models of evolution of galaxies such as PEGASE (Fioc M. \& Rocca-Volmerange B. 1999), LePHARE (Arnouts S. et al. 1999) and GALEV (Kotulla R. et al. 2009) in order to estimate photometric redshifts of near located and all field objects. 


\section{References}

Arnouts, S., Cristiani, S., Moscardini, L., et al., 1999, MNRAS, 310, 540

Fioc, M., \& Rocca-Volmerange, B., 1997, A\&A, 326, 950

Franceschini, A., Vercellone, S., \& Fabian, A.C., 1998, MNRAS, 297, 817

Goss, W.M., Parijskij, Yu.N., Soboleva, N.S., et al., 1992, AstRep, 69, 673

Kopylov, A.I., Goss W.M., Parijskij, Yu.N., et al., 2006, AstL, 32, 433

Kotulla, R., Fritze, U., Weilbacher, P., et al., 2009, MNRAS, 396, 463

Parijskij, Yu.N., Zhelenkova, O.P., Kopylov, A.I., et al., 2010, PoS 10th EVN Symposium, id. 31

Parijskij, Yu.N., Goss, W.M., Kopylov, A.I., et al., 1996, Bull. SAO, 40, 5

Parijskij, Yu.N., Goss, W.M., Kopylov, A.I., et al., 2000, A\&A Trans., 19, 297 